EBrokqutihewte Ac6ess preview-only content

Advances in Differential Equations and Applications

SEMA SIMAI Springer Series Volume 4, 2014, pp 169-182

Date: 11 Oct 2014

\title{
Functional Output-Controllability of Time-Invariant Singular Linear Systems
}

Abstract

In the space of finite-dimensional singular linear continuous-time-invariant systems described in the form 1

$E \dot{x}(t)=A x(t)+B u(t)$

$y(t)=C x(t)$

where

$E, A \in M=M_{n}(\mathrm{C})$,

$B \in M_{n \times m}(\mathrm{C})$,

$C \in M_{p \times n}(\mathrm{C})$, functional output-controllability character is considered. A simple test based in the computation of the rank of a certain constant matrix that can be associated to the system is presented.

Page $\% \mathrm{P}$

Page 1 


\title{
Functional Output-Controllability of Time-Invariant Singular Linear Systems
}

\author{
María Isabel García-Planas and Sonia Tarragona
}

Abstract In the space of finite-dimensional singular linear continuous-timeinvariant systems described in the form

$$
\left.\begin{array}{rl}
E \dot{x}(t) & =A x(t)+B u(t) \\
y(t) & =C x(t)
\end{array}\right\}
$$

where $E, A \in M=M_{n}(\mathbb{C}), B \in M_{n \times m}(\mathbb{C}), C \in M_{p \times n}(\mathbb{C})$, functional outputcontrollability character is considered. A simple test based in the computation of the rank of a certain constant matrix that can be associated to the system is presented.

\section{Introduction}

A great many physical problems as for example electrical networks, multibody systems, chemical engineering, Economics, semidiscretized Stokes equations, Convolutional codes among others, use state space representation as (1) for description.

This linear system can be described with a input-output relation called transfer function obtained by applying Laplace transformation to Eq. (1)

$$
\left.\begin{array}{rl}
s E X-x(0) & =A X+B U \\
Y & =C X,
\end{array}\right\},
$$

obtaining the following relation

$$
H(s) U(s)=C(s E-A)^{-1} x(0)+C(s E-A)^{-1} B U(s) .
$$

\section{M.I. García-Planas ( $₫)$}

Universitat Politècnica de Catalunya, Barcelona, Spain

e-mail: maria.isabel.garcia@upc.edu

\section{S. Tarragona}

Universidad de León, León, Spain

e-mail: sonia.tarragona@unileon.es 

1. Cardetti, F., Gordina, M.: A note on local controllability on lie groups. Syst. Control Lett. 57, 978-979 (2008) CrossRef

2. Chen, C.: Introduction to Linear System Theory. Holt, New York (1970)

3. Dai, L.: Singular Control Systems. Springer, New York (1989) CrossRef

4. Díaz, A.: Sistemas Singulares. Invariantes y Formas Canónicas. PhD. Thesis, Universitat Politècnica de Catalunya (2006)

5. Diaz, A., Garcia-Planas, M.I.: An alternative collection of structural invariants for matrix pencils under strict equivalence. WSEAS Trans. Syst. Control 4(10), 487-496 (2009)

6. Domínguez-García, J.L.: Computing bounds for the distance of functional output-controllable systems representing fixed speed wind turbine. Cybern. Phys. 2(2), 77-83 (2013)

7. Domínguez-García, J.L., García-Planas, M.I.: Output controllability analysis of fixed speed wind turbine. In: Proceedings of the 5th International Conference on Physics and Control, León (2011)

8. Ferreira, P.: On degenerate systems. Int. J. Control 24(4), 585-588 (1876) CrossRef

9. Fragouli, Ch., Wesel, R.D.: Convolutional Codes and Matrix Control Theory. In: Proceedings of the 7th International Conference on Advances in Communications and Control, Athens (1999)

10. García-Planas, M.I., Domínguez-García, J.: Alternative tests for functional and pointwise output-controllability of linear time-invariant systems. Syst. Control Lett. 62(5), 382-387 (2013) CrossRef

11. García-Planas, M.I., Tarragona, S.: Testing functional output-controllability of time-invariant singular linear systems. Cybern. Phys. 2(2), 957-965 (2013)

12. García-Planas, M.I., Tarragona, S.: Analysis of functional output-controllability of time-invariant singular linear systems. In: Proceedings of the XXIII Congreso de Ecuaciones Diferenciales y Aplicaciones / XIII Congreso de Matemática Aplicada, Castellón 2013.e-Treballs d'Informàtica i Tecnologia, N. 15, pp. 957-965, Publicacions de la Universitat Jaume I (2014).

13. Germani, A., Monaco, S.: Functional output-controllability for linear systems on hilbert. Syst. Control Lett. 2(5), 313-320 (1983) CrossRef

14. Kundur, K.: Power System Stability and Control. McGraw-Hill, New York (1994)

\section{About this Chapter}

Title

Functional Output-Controllability of Time-Invariant Singular Linear Systems

Book Title

Advances in Differential Equations and Applications

Book Part

Part III

Pages

Copyright

pp 169-182

2014

DOI

Print ISBN

10.1007/978-3-319-06953-1_17

978-3-319-06952-4

Online ISBN

978-3-319-06953-1

Series Title

SEMA SIMAI Springer Series

Series Volume

4

Series ISSN

2199-3041

Publisher

Springer International Publishing

Copyright Holder

Springer International Publishing Switzerland 
- About this Book

Topics

- Ordinary Differential Equations

- Partial Differential Equations

- Numerical Analysis

- Dynamical Systems and Ergodic Theory

Industry Sectors

- Oil, Gas \& Geosciences

eBook Packages

- eBook Package english full Collection

- eBook Package english Mathematics

Editors

- Fernando Casas $\square^{(11)}$

- Vicente Martínez ${ }^{(12)}$

Editor Affiliations

- 11. Dept. de Mathemàtiques and IMAC, Universitat Jaume I

- 12. Dept. de Mathemàtiques and IMAC, Universitat Jaume I

Authors

- María Isabel García-Planas ${ }^{(13)}$

- Sonia Tarragona ${ }^{(14)}$

Author Affiliations

- 13. Universitat Politècnica de Catalunya, Barcelona, Spain

- 14. Universidad de León, León, Spain

Continue reading...

To view the rest of this content please follow the download PDF link above.

Over 8.5 million scientific documents at your fingertips

(C) Springer, Part of Springer Science+Business Media 
Let your

journey

begin with

Springer...

悬 Springer 